\title{
5G Wireless/Wired Convergence of UFMC Based Modulation for Intensity Modulation Direct Detection
}

\author{
Keerthivasan $\mathrm{K}^{\mathrm{a}, 1}$, Shibu $\mathrm{S}^{\mathrm{b}}$ \\ ${ }^{a} P G$ Scholar, Dept of ECE, Panimalar Engineering College, Chennai, India \\ ${ }^{b}$ Assistant Professor, Dept of ECE, Panimalar Engineering College, Chennai, India
}

\begin{abstract}
Faster data speeds, shorter end-to-end latencies, improved end-user service efficiency, and a wider range of multi-media applications are expected with the new $5 \mathrm{G}$ wireless services. The dramatic increase in the number of base stations required to meet these criteria, which undermines the low-cost constraints imposed by operators, demonstrates the need for a paradigm shift in modern network architecture. Alternative formats will be required for next-generation architectures, where simplicity is the primary goal. The number of connections is expected to increase rapidly, breaking the inherent complexity of traditional coherent solutions and lowering the resulting cost percentage. A novel implementation model is used to migrate complex-nature modulation structures in a highly efficient and cost-effective manner. Theoretical work to analyses modulations' behavior over a wired/fiber setup and wireless mode is also provided. The state-of-the-art computational complexity, simplicity, and ease of execution while maintaining efficiency throughput and bit error rate.
\end{abstract}

Keywords: 4G, 5G, OFDM, Modulation, UFMC, Intensity Modulation Direct Detection.

\section{Introduction}

As the research community focuses on the next generation requirements of high data rate, low latency, low energy consumption, high scalability, improved connectivity, reliability, and higher security, it will become clearer how future architectures will need to be designed to meet these challenges A key factor for mobile operators is the rapid increase in mobile applications that require low network latency, which has resulted in a market shift. To reduce network latency, all telecommunications equipment vendors now have plans to evolve their network technologies. This latency is caused by the wireless in which communication link's unreal ability due to time-varying channel fading and multiple propagation paths. The key to achieving low latency at the physical layer is to use the right technologies to overcome wireless channels' limitations. Synchronization, channel coding, interleaving,

${ }^{1}$ Keerthivasan K, PG Scholar, Dept of ECE, Panimalar Engineering College, Chennai, India, Email:keerthivasan.k21@gmail.com 
demodulation, channel estimation, multiple input, multiple output detection, and other techniques are used in baseband systems. Many redundancies are introduced when channel estimation is used in a multi-antenna receiver system.

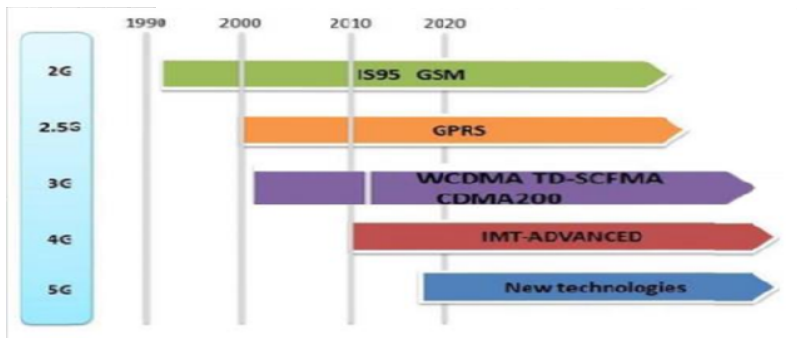

Figure 1. Evolution of mobile networks

Orthogonal Frequency Division is a technique for dividing frequencies that are orthogonal to Although the multiplexing technique has established itself as the dominant modulation scheme in the fourth generation of broadband systems, some of its shortcomings have prompted the research community to consider other possible alternatives that could improve overall performance in the next generation [5-6].

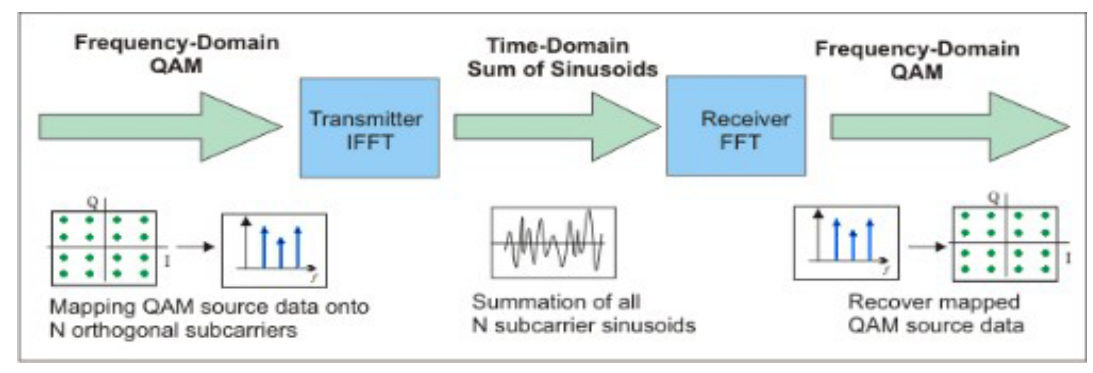

Figure 2. OFDM General blocks

Inter-carrier interference should be avoided by spacing the sub-carriers evenly. Figure 2 depicts an OFDM multicarrier modulation model. One method is to keep them apart by stationing ominous guard bands among them. We'll focus on three of them in this paper because they've gotten a lot of attention recently: Filter Bank Multi-Carrier (FBMC), Universal-Filtered Multi-Carrier (UFMC), and Generalized Frequency Division Multiplexing are all examples of frequency division multiplexing (GFDM). All of them have arisen to address some of the shortcomings of OFDM, and it is this fact, as well as the properties that follow it, that will make them suitable for specific applications.

\section{Proposed Method}

\subsection{Signal carrier}

Signal carrier waveforms have a lower peak-to-average power ratio, resulting in increased power amplifier efficiency and battery life. Furthermore, the amplifier is required in the presence of a multipath to achieve high spectral efficiency. Single carrier quadrature amplitude modulation, single-carrier frequency-domain equalization, 
single-carrier frequency division multiplexing, and zero-tail SC-FDM are all examples of single-carrier quadrature amplitude modulation.

\subsection{Multi-Carrier}

Within a given bandwidth, multicarrier OFDM-based waveforms support orthogonal sub- carriers. Furthermore, waveforms based on multicarrier OFDM are easily integrated with MIMO, resulting in high spectral efficiency. The following are examples of multicarrier waveforms: cyclic prefix OFDM, CP-OFDM with four weighted overlaps, universal-filtered multicarrier, filter bank multicarrier generalized frequency division multiplexing CP-OFDM is efficient because it employs the fast inverse Fourier transform in both the transmitter and receiver.

\section{$2.3 \mathrm{Mm}$-Wave}

The mm-wave band provides $5 \mathrm{G}$ systems with a Gigahertz amount of bandwidth. The following are some of the ramifications of using the mm-wave spectrum. The ability to share the spectrum with satellite or radar systems using cognitive radio techniques. It is possible to generate very narrow beams with smaller directional and adaptive antenna arrays because of the small wavelength. Mm-wave can deliver gigabit-per-second peak, average, and outage rates required in various $5 \mathrm{G}$ scenarios.

\subsection{Massive MIMO}

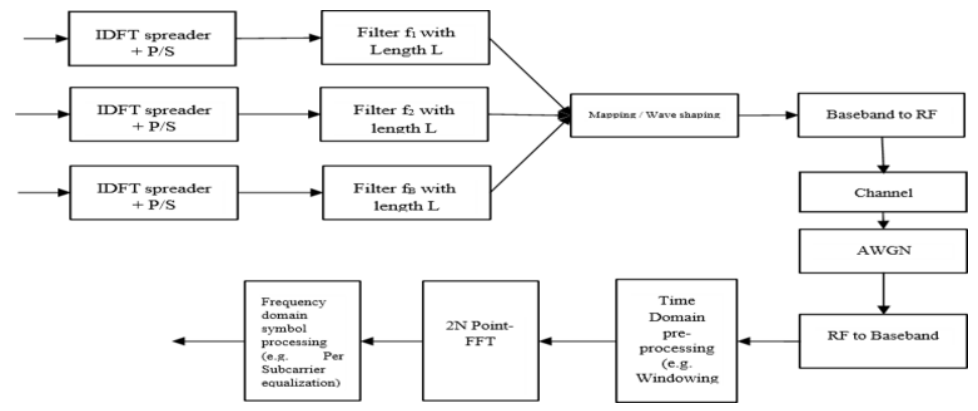

Figure 4. Proposed Method

All negative subcarrier records in the primary preparing image have invalid tones, and all positive subcarrier records in the subsequent image have invalid tones.To characterize our UFMC system, consider a simple one-to-one transmission. The total $\mathrm{N}$ subcarriers are divided into B sub-bands. It is worth mentioning that if the order of the filter $\mathrm{L}=1$, UFMC converges to ZP-OFDM. Figure 4 shows that the block diagram at the Transmitter and Receiver. By simulating a simple point-to-point UFMC transceiver, we will see the spectrum of a UFMC signal. Our transmission parameters will be the same as they were in the case of OFDM and f-OFDM. Below table 1 lists all the parameters that were used. 
Table 1. Simulation Parameters UFMC transceiver

\begin{tabular}{lc}
\hline Parameter & Wired/Wireless standard \\
\hline FFT/IFFTsize & 1024 \\
Numberofuseddatasubcarriers & 360 \\
Numberofpilotsubcarriers & 12 \\
Numberofguardbands & 32 \\
ZeroPadding & $1 / 8$ \\
ChannelBandwidth(MHz) & 5 \\
Modulation & QAM \\
\hline
\end{tabular}

\section{Results and Discussion}

Based on the simulation results, the proposed method offers more significant improvements. The coverage areas of femto cells may overlap in a deployment where a certain number of femto cells are deployed. Data is transferred orthogonally to each node in this network. Table 2 shows the summary of the existing and proposed methods. It demonstrates that quadrature amplitude modulation can be used to estimate network path loss.

Table 2. Summary

\begin{tabular}{lll}
\hline Description & Existing & Proposed \\
\hline Modulation factor & PAM & QAM \\
PAPR & $5.4396[8]$ & 4.516 \\
\hline
\end{tabular}
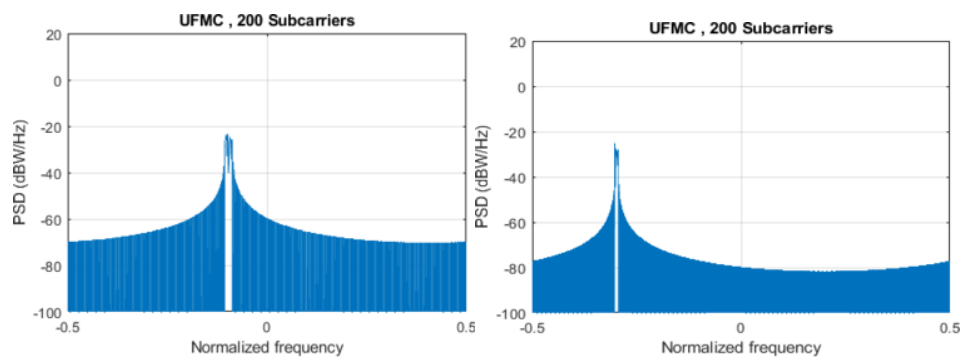

Figure 5. UFMC subcarriers for FFT size 512 (Left) and FFTsize1024(Right)

The reduction in the power density of an electromagnetic wave propagates through space, known as path loss or path attenuation. Path loss is an important factor to consider when distances shows that path loss leads to increases in SNR. Free-space loss, refraction, diffraction, reflection, aperture-medium coupling loss, and absorption 
are all path loss examples using wireless cellular networks to their full potential. Figure 5shows the UFMC subcarriers for FFT size 512 (Left) and FFTsize1024 (Right). It means that as the likelihood of detection rises, the likelihood of a false alarm decreases. The size of the FFT is 1024 bytes, and the number of subcarriers is two. For each modulation technique (16 QAM and 64 QAM), values are taken. The 64 QAM modulation technique yields the best results. The size of the FFT is 512 bytes, and the number of subcarriers is two. For each modulation technique (16 QAM and 64 QAM), values are taken. The 64 QAM modulation technique yields the best results.

\section{Conclusion}

Amplitude Modulation-Direct Detection Optical Front Hauling has been proposed to solve the next generation of $5 \mathrm{G}$ cellular networks. The various waveform candidates from wireless radio access networks have been directly converted to fiber front hauls and tested. State-of- the-art computational sophistication and performance while retaining the critical low out-of- band radiation and high data speeds of each new modulation in greater scalability and lower cost. The evaluating OFDM-based modulation schemes for future wireless communication. UFMC was one of the modulation schemes we considered. We evaluated the performance of each modulation scheme in both synchronous and asynchronous modes.

\section{References}

[1] Sarmiento Hernández, S., 2020. Design and optimization of metro-access networks supporting 5gservices.

[2] Dahawi, T.H., Yusoff, Z., Salleh, M.S. and Senior, J.M., 2021. Low-cost MIMO-RoF- PON architecture for next-generation integrated wired and wireless access networks. Journal of Optical Communications and Networking, 13(3),pp.41-52.

[3] Elmagzoub, M.A., Shaikh, A., Alghamdi, A. and Rajab, K., 2020. A Review on MIMO Wireless Signals over Fibre for Next Generation Fibre Wireless (FiWi) Broadband Networks. Electronics, 9(12),p.2014.

[4] Karembera, R., Nfanyana, K. and Gibbon, T., 2021. Simultaneous transmission of clock and data signals in photonic-assisted WDM passive optical networks. JOSA B, 38(3), pp.914-921.

[5] Dayana, R., Malarvezhi, P., Vadivukkarasi, K. and Kumar, R., 2020. UF0MC-IOTA Based Cognitive Radio Transceiver. Wireless Personal Communications, 114, pp.2105-2119.

[6] Nagarajan, N.R. and Maheswari, M., 2020, December. A survey on UFMC filter designs for 5G M2M. In Journal of Physics: Conference Series (Vol. 1706, No. 1, p. 012158). IOP Publishing.

[7] Zhang, X., Wang, X. and Chen, Z., 2018, May. A transceiver modification for a universal filtered multicarrier scheme in a 5G cellular network. In 2018 IEEE International Conference on Communications (ICC) (pp. 1-6). IEEE.

[8] Kishore, K.K., Umar, P.R. and Naveen, V.J., 2017. Comprehensive analysis of UFMC with OFDM and FBMC. Indian Journal of Science and Technology, 10(17),pp.1-7

[9] Thiyagarajan, S. and Veerappan, S., Performance Analysis of UFMC and its Comparison withCPOFDM.

[10] Salah, S., Selem, M., Seleem, H. and Ali, M.A., 2020. Reduced OoB Emission and PAPR Using Partial-OSLM technique in 5G UFMC Systems. Journal of Engineering Research, 4(December),pp.16.

[11] Keerthivasan K, and Shibu S .Survey on Mobile Edge Computing for the Internet of ThingsSystem .(doi:10.3233/APC200191) 